\title{
Coupled delta-beta wave activity might predict social anxiety in children
}

\author{
By Dr Jessica Edwards
}

Oscillations in frontal brain activity can be categorised as slow (delta) or fast (beta) waves that have different functional and behavioural correlates. Recording these waves by electroencephalography (EEG) has shown that coupled delta-wave and beta-wave oscillations might be a correlate of higher neuroendocrine (namely cortisol) activity and social anxiety in adults; ${ }^{1}$ whether this is the case in children, however, is unclear. Now, researchers from McMaster University, Canada, have examined whether individual differences in salivary cortisol levels at baseline and parent-reported social anxiety levels are associated with resting, coupled delta-beta frontal wave activity.

They collected EEG recordings from 50 children with a mean age of 7.59 years and collated the data with basal salivary cortisol and social anxiety levels collected at two time points, separated by 1 year. They found that stably high basal salivary cortisol levels and social anxiety across the two time-points, were independently associated with relatively high, correlated delta-betawave activity.

The researchers propose that such neural oscillatory patterns may help identify children at risk for stable avoidance and fear-related profiles. They consider that future studies should extend such longitudinal analyses of coupled delta-beta-wave activity, starting from early childhood to better understand the value of coupled delta-beta-wave activity in predicting social and emotional development in children. Furthermore, work is needed to explore the future potential of psychological interventions on these correlations to reduce social anxiety in both adults and children.

\section{References:}

Schmidt, L.A. and Poole, K.L. (2019), Frontal brain delta-beta correlation, salivary cortisol, and social anxiety in children. J. Child Psychol. Psychiatr. 60: 646-654. doi: 10.1111/jcpp.13016.

\section{Further reading:}

${ }^{1}$ Miskovic V. et al. (2010), Frontal brain oscillations and social anxiety: a cross-frequency spectral analysis during baseline and speech anticipation. Biol Psychol. 83:125-132. doi: 10.1016/j. biopsycho.2009.11.010.

\section{Glossary:}

Electroencephalography (EEG): a non-invasive, electrophysiological method to record electrical activity generated by synchronised neurons in the brain.

Cortisol: a glucocorticoid-class steroid hormone made in the adrenal gland and controlled by the hypothalamus, pituitary gland and adrenal gland. Often referred to as the "stress hormone", cortisol is typically released during stress, but also helps regulate blood sugar levels, metabolism and inflammation.

Delta wave: a high-amplitude wave produced in the brain, with a low oscillation frequency of $\sim 0.5-$ 4 hertz. Delta-wave activity is typically associated with stage 3 NREM sleep.

Beta wave: a low-amplitude wave produced in the brain, with a high oscillation frequency of $\sim 12.5-30$ hertz. Beta wave activity is typically associated with wakefulness. 